Psychotherapeut 2022 $67: 313-319$ https://doi.org/10.1007/s00278-021-00558-7

Angenommen: 5. November 2021

Online publiziert: 10. Dezember 2021

(c) Der/die Autor(en) 2021

\section{Einstellung der \\ Allgemeinbevölkerung zur Schweigepflicht von Ärzt:innen und Psychotherapeut:innen bei Patient:innen mit extremistischer Einstellung}

Thea Rau · Sophia Mayer · Jörg M. Fegert · Marc Allroggen

Klinik für Kinder- und Jugendpsychiatrie/Psychotherapie, Universitätsklinikum Ulm, Ulm, Deutschland

\title{
Zusammenfassung
}

Hintergrund: Um extremistische Gewalttaten zu verhindern, wurde in der Vergangenheit immer wieder diskutiert, inwieweit eine allgemeine Aufhebung der ärztlichen Schweigepflicht und der Verschwiegenheitspflichten bei psychologischen Psychotherapeut:innen und Ärzt:innen im Zusammenhang mit extremistischen Einstellungen bei Patient:innen ein sinnvolles Mittel zur Gefahrenabwehr sei. Bislang gibt es keine Untersuchung darüber, ob dies im politischen Diskurs ein mehrheitsfähiges Argument ist, da es keine Befragung zur Einstellung der Allgemeinbevölkerung im Zusammenhang mit religiösem oder politischem Extremismus und Verschwiegenheitspflichten gibt.

Material und Methoden: Anhand einer bevölkerungsrepräsentativen Befragung ( $n=2503)$ wurde die Einstellung der Allgemeinbevölkerung untersucht.

Ergebnisse: Es zeigt sich, dass über die Hälfte der Befragten (58\%) den Schutz vor möglicher Gefährdung durch extremistische Gewalt als wichtiger ansehen als die Einhaltung der Schweigepflicht bei Psychiater:innen und Psychotherapeut:innen. Über die Hälfte (54\%) stimmen dem zu, dass die Möglichkeit zum Bruch der Schweigepflicht grundsätzlich bestehen sollte, wenn Psychotherapeut:innen oder Psychiater:innen von extremistischen Einstellungen bei Patient:innen erfahren. Die Zustimmung war insbesondere dann höher, wenn die Befragten Terrorismus grundsätzlich in einen Zusammenhang mit psychischen Erkrankungen brachten.

Schlussfolgerung: Die Ergebnisse zeigen einerseits die Notwendigkeit, über die Bedeutung der Schweigepflicht als zentrale Grundlage einer Heilbehandlung stärker aufzuklären, andererseits aber auch sehr kritisch Stellung zu beziehen, welche Rolle psychische Erkrankungen tatsächlich bei der Entstehung von extremistischer Gewalt spielen, um eine Stigmatisierung von Betroffenen zu vermeiden.

\section{Schlüsselwörter}

Terrorismus · Psychische Erkrankungen · Behandler-Patient-Beziehungen · Stigmatisierung . Bevölkerungsrepräsentative Befragung

Die Schweigepflicht für Ärzt:innen und psychologische Psychotherapeut:innen ist ein aktuelles Thema in der Diskussion um die Verhinderung von extremistischen Straftaten, insbesondere im Zusammenhang mit psychisch auffälligen Personen. In der vorliegenden Studie wird erstmalig untersucht, wie die Allgemeinbevölkerung den Umgang mit der Schweigepflicht im Zusammenhang mit religiösem oder politischem Extremismus sieht. 


\section{Einleitung}

Das Attentat im Juni 2021 in Würzburg mit mutmaßlich extremistischem Hintergrund entfachte erneut die Debatte über die Möglichkeiten der Verhinderung von Gewalttaten durch eine psychiatrische Behandlung (taz.de 2021). Bereits 2016 wurden im Zusammenhang mit Attentaten und in der Folge der Verschärfung des Antiterrorgesetzes eine Beteiligung von Ärzt:innen und Psychotherapeut:innen an der Terrorbekämpfung gefordert und auch die Lockerung der Schweigepflicht diskutiert (tagesschau.de 2020; Linden und Helmchen 2018; dw.com 2020). Kammern, insbesondere die Psychotherapeutenkammer, und Fachgesellschaften der Heilberufe lehnten allerdings in der Vergangenheit eine Lockerung der bestehenden Schweigepflicht (bundesaerztekammer.de 2021; bptk.de 2019) und Reform der Gesetzesgrundlage vehement $a b$. Sie warnten davor, sich wegen der angespannten Sicherheitslage zu vorschnellen politischen oder gar rechtlichen Änderungen verleiten zu lassen (bayerische-staatszeitung.de 2021; aerzteblatt.de 2020; psychotherapeutenjournal.de 2015). Neben dem Hinweis auf bereits hinreichend bestehende Möglichkeiten, die Schweigepflicht bei konkreter Gefahr zu brechen, wurde v.a. auf die negativen Folgen einer grundsätzlichen Lockerung der Schweigepflicht für das Vertrauensverhältnis zwischen Patient:innen und Berufsgeheimnisträgern, z.B. Ärzt:innen und Psychotherapeut:innen, hingewiesen. Dies sei insbesondere relevant für Patient:innen, bei denen aggressive Fantasien im Rahmen der Behandlung kommuniziert werden (Klett und Tessmer 2016). Die Diskussion über die Schweigepflicht führte zudem dazu, dass extremistische Gewalt in der Öffentlichkeit primär mit dem Vorliegen psychischer Störungen in Verbindung gebracht wurde (tagesschau.de 2020; sueddeutsche.de 2021). Diese Annahme lässt sich wissenschaftlich nicht begründen und kann zu einer allgemeinen Stigmatisierung von psychisch kranken Menschen führen (Allroggen 2020; Bühring 2016, 2018; Dom et al. 2018; Schomerus et al. 2017).

Betrachtet man die Schweigepflicht von Ärzt:innen und psychologischen Psy- chotherapeut:innen in Bezug auf das Arzt-Patienten-Verhältnis, stellt diese eine "Grundvoraussetzung ärztlichen Wirkens" dar. Die Schweigepflicht zählt zu den höchsten Berufs- und Standespflichten von Ärzt:innen (Parzeller et al. 2005) und psychologischen Psychotherapeut:innen (bptk.de 2019). Das gesetzliche Gerüst zur Verschwiegenheitspflicht im Rahmen einer Behandlung wurzelt im allgemeinen Persönlichkeitsrecht (Art. 1 Abs. 1 GG i. V.m. Art. 2 Abs. 1 GG) bzw. dem Recht auf informationelle Selbstbestimmung. Die Weitergabe von Informationen durch Angehörige von Heilberufen setzt demnach eine, ggf. auch mutmaßliche, Einwilligung der Patientin/des Patienten voraus. Gegen den Willen oder ohne Einverständnis von Patient:innen ist demgegenüber eine Weitergabe von Informationen nur unter engen gesetzlichen Voraussetzungen möglich, z.B. bei Vorliegen eines rechtfertigenden Notstands ( $§ 34$ StGB) oder im Fall einer möglichen Kindeswohlgefährdung ( $\$ 4$ KKG). Eine Verpflichtung zur Weitergabe besteht z.B. im Rahmen des Infektionsschutzgesetzes oder wenn Ärzt:innen bzw. Psychotherapeut:innen glaubhaft von der Planung einer schweren Straftat nach dem Katalog des $§ 138$ StGB erfahren.

Wenig Beachtung fand in der bisherigen kontroversen Auseinandersetzung zwischen Fachgesellschaften, Kammern, Gesetzgeber und Regierung (aerztezeitung.de 2021; handelsblatt.com 2021), ob die vonseiten der Politik geforderte Lockerung der Schweigepflicht ein mehrheitsfähiges Argument ist, mit dem sich die ärztliche und psychotherapeutische Profession im Sinne von Aufklärung über die Bedeutung der Schweigepflicht auseinandersetzen muss. Es ist aus Sicht von Ärzt:innen und Psychotherapeut:innen im Zusammenhang mit ihrer Tätigkeit aber ein entscheidender Faktor, ob die zentrale Bedeutung der Schweigepflicht von Patient:innen und der Bevölkerung wahrgenommen wird. Denn nur, wenn hierüber eine prinzipielle Verständigung besteht, kann der Schutz- und Vertrauensraum im Rahmen einer Behandlung überhaupt wirksam sein. Daher ist es das Ziel der folgenden Untersuchung, die Allgemeinbevölkerung nach ihrer Einstellung zu befragen, ob sie eine Lockerung der Schweigepflicht von Psychiater:innen und Psychotherapeut:innen im Zusammenhang mit Extremismus befürworten würden. Zudem soll untersucht werden, ob die Befürwortung einer Lockerung der Schweigepflicht eine Korrelation mit der Überzeugung zeigt, dass extremistische Gewalttäter:innen unter psychischen Störungen leiden, oder ob diese von einer politischen Orientierung abhängt, und welche Rolle speziell Psychotherapeut:innen und Psychiater:innen in der Prävention von extremistischen Taten zugeschrieben wird.

\section{Methode}

Zur Beantwortung der Fragestellungen wurde nach dem "Random-route"-Verfahren mit Startadressenvorgabe eine für Deutschland in Bezug auf Alter, Geschlecht, Bildung und Wohnregion repräsentative Stichprobe gezogen. In diesen insgesamt 5668 ermittelten Haushalten wurden Interviewpartner:innen nach dem Zufallsprinzip ("Schwedenschlüssel“) ausgewählt. Teilnehmen konnten grundsätzlich Personen mit einem Mindestalter von 14 Jahren sowie mit ausreichenden Deutschkenntnissen. Die Teilnahme an der Studie war freiwillig; An der Untersuchung nahmen 2524 Haushalte teil; diese wurden im Zeitraum von Februar 2020 bis April 2020 für ein persönliches Interview kontaktiert. Eine Einwilligung zur Studienteilnahme wurde schriftlich erhoben.

Zu Beginn des Interviews erhob die/der Studienmitarbeiter:in die soziodemografischen Daten von der Zielperson des Haushalts. Anschließend erhielt diese einen Fragebogen sowie einen Umschlag, in dem sie den ausgefüllten Fragebogen später selbst verschließen sollte. Die/der Interviewer:in konnte bei Verständnisproblemen um Hilfe gebeten werden. Die Befragung wurde von dem deutschen Markt- und Sozialforschungsinstitut USUMA (Unabhängiger Service für Umfragen, Methoden und Analysen), Berlin-Weißensee, durchgeführt und enthielt insgesamt 119 Fragen $z u$ unterschiedlichen Themen wie zur politischen Einstellung, zu belastenden Kindheitserfahrungen und psychosozialen Belastungen. In dieser Arbeit wird nur ein Teil der Fragen berichtet. 
Tab. 1 Soziodemografische Daten der Studienteilnehmenden

\begin{tabular}{|c|c|}
\hline & $\begin{array}{l}\text { Anzahl (Anteil, } \\
\text { gültige \%) }\end{array}$ \\
\hline \multicolumn{2}{|l|}{ Geschlecht } \\
\hline Weiblich & $1256(50)$ \\
\hline Männlich & $1247(50)$ \\
\hline \multicolumn{2}{|l|}{ Höchster Schulabschluss } \\
\hline Ohne & $52(2)$ \\
\hline Haupt-/Volksschule & $702(28)$ \\
\hline Mittlere Reife & $805(32)$ \\
\hline $\begin{array}{l}\text { Polytechnische Ober- } \\
\text { schule/Fachschule }\end{array}$ & $307(12)$ \\
\hline Hochschulreife/Abitur & $340(14)$ \\
\hline $\begin{array}{l}\text { Fachhochschul- oder } \\
\text { Universitätsabschluss }\end{array}$ & $258(10)$ \\
\hline Schüler*in & $37(1)$ \\
\hline \multicolumn{2}{|c|}{ Haushaltseinkommen (monatlich) } \\
\hline Kein Einkommen & $111(4)$ \\
\hline$<1250 €$ & $828(33)$ \\
\hline Bis $2500 €$ & $891(36)$ \\
\hline$>2500 €$ & $275(11)$ \\
\hline Gesamt & $2503(100)$ \\
\hline
\end{tabular}

Die Untersuchung erfolgte in Übereinstimmung mit der Deklaration von Helsinki (Weltärztebund 2013), sie erfüllt die ethischen Richtlinien des International Code of Marketing and Social Research Practice der International Chamber of Commerce und der European Society for Opinion and Marketing Research (ICC/ESOMAR 2007) und erhielt ein positives Votum durch die Ethikkommission der Universität Leipzig.

\section{Stichprobenbeschreibung}

Insgesamt wurden 2503 Personen in die Studie eingeschlossen. Nicht verwertbar waren 21 Interviews und wurden daher für weitere Auswertungen aus dem Datensatz genommen. Das Durchschnittsalter der Teilnehmenden betrug 49,52 Jahre (SD $\pm 17,51$ Jahre), 50,2\% waren weiblich (für detaillierte Informationen zu den Studienteilnehmenden: - Tab. 1).

\section{Fragestellungen}

Zur Beantwortung der Fragestellung wurden den Studienteilnehmenden 3 Fragen vorgelegt, die die Einstellung zum Bruch der Schweigepflicht im Zusammenhang mit extremistischer Einstellung und Gewalt auf einer 5 -stufigen Skala erfassten

Tab. 2 Einstellung zum Bruch der ärztlichen Schweigepflicht im Zusammenhang mit extremistischen Einstellungen von Patient:innen und zu psychiatrischen/psychotherapeutischen Behandlung von Extremist:innen

(Skala $1={ }_{\text {"stimme nicht }} \mathrm{zu}$ " bis $5={ }_{\text {"stimme }} \mathrm{zu}$ ")

\begin{tabular}{|l|l|l|l|l|l|l|}
\hline 1 & 2 & 3 & 4 & 5 & Gesamt & $\begin{array}{l}\text { Keine } \\
\text { Angabe }\end{array}$ \\
\hline
\end{tabular}

Absolute Häufigkeit (gültige Prozent)

1. Ärzte und Psychotherapeuten sollten grundsätzlich ihre Schweigepflicht brechen dürfen, wenn sie von extremistischen politischen oder religiösen (z. B. islamistischen) Einstellungen ihrer Patienten erfahren

\begin{tabular}{|l|l|l|l|l|l|l|}
$351(14,1)$ & $248(10,0)$ & $563(22,6)$ & $538(21,6)$ & $790(31,7)$ & 2490 & 13 \\
\hline
\end{tabular}

2. Polizei und Staatsanwaltschaft sollten grundsätzlich Einsicht in eine Patientenakte von Patienten mit extremistischen politischen oder religiösen (z. B. islamistischen) Einstellungen nehmen dürfen

\begin{tabular}{|l|l|l|l|l|l|l|}
$397(12,3)$ & $261(10,4)$ & $586(23,4)$ & $540(21,6)$ & $805(32,3)$ & 2499 & 4
\end{tabular}

3. Der Schutz vor möglichen Gefährdungen durch politische oder religiöse extremistische Gewalt ist wichtiger als die Einhaltung der ärztlichen Schweigepflicht

\begin{tabular}{|l|l|l|l|l|l|l|}
$254(10,2)$ & $225(9,0)$ & $584(23,4)$ & $573(22,9)$ & $865(34,6)$ & 2501 & 2
\end{tabular}

4. Menschen, die einen Terrorakt planen oder durchführen, sind meistens psychisch krank

\begin{tabular}{|l|l|l|l|l|l|l|}
\hline $367(14,8)$ & $336(13,5)$ & $606(24,4)$ & $506(20,4)$ & $665(26,8)$ & 2480 & 23
\end{tabular}

5. Personen mit extremistischen politischen oder religiösen (z. B. islamistischen) Einstellungen haben häufig ein psychisches Problem

\begin{tabular}{|l|l|l|l|l|l|l|}
\hline $328(13,2)$ & $350(14,1)$ & $620(24,9)$ & $536(21,5)$ & $655(26,3)$ & 2489 & 14
\end{tabular}

6. Für Menschen, die einen Terrorakt geplant oder durchgeführt haben, sollte eine psychotherapeutische bzw. psychiatrische Behandlung Pflicht sein, wenn sie gefasst werden

\begin{tabular}{|l|l|l|l|l|l|l|}
$394(15,8)$ & $273(11,0)$ & $546(21,9)$ & $469(18,8)$ & $809(32,5)$ & 2491 & 12
\end{tabular}

7. Menschen mit extremistischen Gewaltvorstellungen kann durch eine psychotherapeutische bzw. psychiatrische Behandlung geholfen werden

\begin{tabular}{|l|l|l|l|l|l|l}
\hline $570(22,9)$ & $424(17,1)$ & $748(30,1)$ & $399(16,1)$ & $343(13,8)$ & 2484 & 19
\end{tabular}

("stimmenichtzu“ bis "stimmezu").Zudem wurde anhand von 4 Fragen untersucht, inwieweit ein Zusammenhang zwischen dem Vorliegen psychischer Störungen und Problemen sowie der psychotherapeutischen/psychiatrischen Behandlungsnotwendigkeit bei extremistischer Gewalt besteht (- Tab. 2). Ergänzend wurde die politische Orientierung (links, Mitte, rechts) auf einer 11-stufigen Skala erfasst.

\section{Statistische Analysen}

Für die statistische Datenanalyse wurden die Befragungsdaten in die Auswertungssoftware IBM SPSS Statistics 27 importiert. Die einzelnen Antworten auf einer 5-stufigen Ordinalskala wurden deskriptiv ausgewertet. Aussagen, die mit 4 oder 5 (stimme zu) bewertet wurden, wurden als Zustimmung bewertet. Darüber hinaus wurden bivariate Korrelationen mittels des Pearson-Korrelationskoeffizienten berechnet zur Aussage, inwieweit von den Befragten eine Verbindung zwischen dem Vorliegen psychischer Störungen sowie der psychotherapeutischen/psychiatrischen Behandlungsnotwendigkeit bei extremistischer Gewalt gesehen wird. Zusätzlich wurde untersucht, inwieweit die politische Einstellung einen Einfluss auf das Antwortverhalten hat. Dies wurde mithilfe multivariater Varianzanalysen sowie $t$-Tests und anschließender Post-hocAnalysen getestet. Für die Berechnungen wurden die Fragen zur politischen Orientierung, die auf einer 11-stufigen Skala erfasst wurden, in 3 Kategorien (links: 1-3; Mitte: 4-8 und rechts: 9-11) zusammengefasst.

\section{Ergebnisse}

Der Aussage, dass Psychiater:innen und Psychotherapeut:innen grundsätzlich ihre Schweigepflicht sollten brechen dürfen, wenn sie von extremistischen Einstellungen erfahren, stimmen $53,3 \%(n=1328)$ der Befragten überwiegend zu. Auch die Aussage, dass Polizei und Staatsanwaltschaft grundsätzlich ein Einsichtsrecht haben sollten, wenn bei Patient:innen eine 


\section{Originalien}

Tab. 3 Zusammenhang der Einstellung zum Bruch der ärztlichen Schweigepflicht mit der Einstellung zu psychischen Erkrankungen von Menschen mit extremistischer Einstellung

\begin{tabular}{|c|c|c|c|}
\hline & $\begin{array}{l}\text { Ärzte und Psychotherapeuten sollten } \\
\text { grundsätzlich ihre Schweigepflicht } \\
\text { brechen dürfen, wenn sie von extre- } \\
\text { mistischen politischen oder religiösen } \\
\text { (z. B. islamistischen) Einstellungen } \\
\text { ihrer Patienten erfahren }\end{array}$ & $\begin{array}{l}\text { Polizei und Staatsanwaltschaft } \\
\text { sollten grundsätzlich Einsicht in } \\
\text { eine Patientenakte von Patienten } \\
\text { mit extremistischen politischen } \\
\text { oder religiösen (z. B. islamistischen) } \\
\text { Einstellungen nehmen dürfen }\end{array}$ & $\begin{array}{l}\text { Der Schutz vor möglichen } \\
\text { Gefährdungen durch politi- } \\
\text { sche oder religiöse extremis- } \\
\text { tische Gewalt ist wichtiger } \\
\text { als die Einhaltung der ärztli- } \\
\text { chen Schweigepflicht }\end{array}$ \\
\hline \multicolumn{4}{|l|}{ Korrelation } \\
\hline $\begin{array}{l}\text { Menschen, die einen Terrorakt } \\
\text { planen oder durchführen, sind } \\
\text { meistens psychisch krank }\end{array}$ & $0,287^{* *}$ & $0,353^{* *}$ & $0,316^{* *}$ \\
\hline $\begin{array}{l}\text { Personen mit extremistischen } \\
\text { politischen oder religiösen (z. B. } \\
\text { islamistischen) Einstellungen } \\
\text { haben häufig ein psychisches } \\
\text { Problem }\end{array}$ & $0,311^{* *}$ & $0,367^{* *}$ & $0,333^{* *}$ \\
\hline $\begin{array}{l}\text { Für Menschen, die einen Terror- } \\
\text { akt geplant oder durchgeführt } \\
\text { haben, sollte eine psychothera- } \\
\text { peutische bzw. psychiatrische } \\
\text { Behandlung Pflicht sein, wenn } \\
\text { sie gefasst werden }\end{array}$ & $0,309^{* *}$ & $0,356^{* *}$ & $0,352^{* *}$ \\
\hline $\begin{array}{l}\text { Menschen mit extremistischen } \\
\text { Gewaltvorstellungen kann } \\
\text { durch eine psychotherapeuti- } \\
\text { sche bzw. psychiatrische Be- } \\
\text { handlung geholfen werden }\end{array}$ & $0,160^{* *}$ & $0,217^{* *}$ & $0,203^{* *}$ \\
\hline
\end{tabular}

\begin{tabular}{|c|c|c|c|}
\hline $\begin{array}{l}\text { Politische Einstel- } \\
\text { lung }\end{array}$ & $\begin{array}{l}\text { Ärzte und Psychotherapeuten sollten } \\
\text { grundsätzlich ihre Schweigepflicht brechen } \\
\text { dürfen, wenn sie von extremistischen poli- } \\
\text { tischen oder religiösen (z. B. islamistischen) } \\
\text { Einstellungen ihrer Patienten erfahren }\end{array}$ & $\begin{array}{l}\text { Polizei und Staatsanwaltschaft sollten } \\
\text { grundsätzlich Einsicht in eine Patien- } \\
\text { tenakte von Patienten mit extremisti- } \\
\text { schen politischen oder religiösen (z. B. } \\
\text { islamistischen) Einstellungen nehmen } \\
\text { dürfen }\end{array}$ & $\begin{array}{l}\text { Der Schutz vor möglichen Ge- } \\
\text { fährdungen durch politische } \\
\text { oder religiöse extremistische } \\
\text { Gewalt ist wichtiger als die Ein- } \\
\text { haltung der ärztlichen Schwei- } \\
\text { gepflicht }\end{array}$ \\
\hline $\begin{array}{l}\text { Linksorientiert; a } \\
n=263\end{array}$ & $3,31(1,51)$ & $3,25(1,48)^{b, c}$ & $3,44(1,43)^{c}$ \\
\hline $\begin{array}{l}\text { Mittig orientiert; } b \\
n=1983\end{array}$ & $3,46(1,36)$ & $3,49(1,33)^{\mathrm{a}, \mathrm{c}}$ & $3,61(1,29)^{c}$ \\
\hline $\begin{array}{l}\text { Rechtsorientiert; c } \\
n=162\end{array}$ & $3,59(1,50)$ & $3,86(1,36)^{a, b}$ & $3,91(1,28)^{a, b}$ \\
\hline Teststatistik & $F(2,2405)=2,270$ & $F(2,2405)=9,950$ & $F(2,2405)=6,413$ \\
\hline$p$-Wert & 0,103 & 0,000 & 0,002 \\
\hline Partielles eta ${ }^{2}$ & 0,002 & 0,008 & 0,005 \\
\hline
\end{tabular}

extremistische Einstellung vorliegt, beantworteten 53,8\% $(n=1345)$ der Befragten zustimmend. Zudem wurde der Schutz vor extremistischer Gewalt von 57,5\% $(n=1438)$ der Befragten als bedeutsamer angesehen als die Schweigepflicht, wohingegen nur ein vergleichsweise geringer Anteil der Befragten von 29,9\%
( $n=742$ ) der Meinung ist, dass Menschen mit extremistischen Gewaltvorstellungen psychotherapeutisch oder psychiatrisch geholfen werden kann (• Tab. 2).

Bei der Betrachtung von Zusammenhängen zeigen sich kleine bis mittlere Korrelationen zwischen der Zustimmung zum Bruch der Schweigepflicht und der Ein- schätzung, dass extremistische Einstellungen und Gewalt mit psychischen Störungen verbunden sind (• Tab. 3).

In der Stichprobe gaben 265 Personen $(10,6 \%)$ an, dass sie eher dem linken, und 163 Personen $(6,5 \%)$, dass sie eher dem rechten politischen Spektrum angehören, während sich 1991 Personen (79,5\%) po- 
litisch in der Mitte einordneten. Beim Vergleich der Einstellungen von politischen Gruppierungen zu bestimmten Fragestellungen verringern sich die Gruppengrößen aufgrund von fehlenden Angaben zu den jeweiligen Fragestellungen gering. Bei der Frage nach einer grundsätzlichen Einsicht von Polizei und Staatsanwaltschaft in Patientenakten bei Patient:innen mit extremistischer Einstellung unterscheiden sich die Einstellungen aller 3 Gruppen. Personen mit eher linker politischer Orientierung stimmen dieser Aussage am wenigsten zu, eher rechtsorientierte Personen am häufigsten (- Tab. 4).

\section{Diskussion}

Die vorliegende Studie untersucht anhand einer bevölkerungsrepräsentativen Stichprobe, inwieweit die Allgemeinbevölkerung den Bruch der Schweigepflicht im Rahmen einer psychiatrischen/ psychotherapeutischen Behandlung im Zusammenhang mit extremistischen politischen oder religiösen Einstellungen von Patient:innen befürwortet. Damit einhergehend wurde untersucht, inwiefern die Bedeutung der Schweigepflicht von Ärzt:innen und Psychotherapeut:innen der Allgemeinbevölkerung präsent ist. Dabei wird deutlich, dass die Mehrheit der Bevölkerung eine Lockerung der Schweigepflicht eher befürworten würde. Diese Einstellung zeigt sich bereits, wenn es lediglich um extremistische Einstellungen (und nicht um drohende Gewalttaten) geht, und ist unabhängig von der politischen Orientierung. Die vorliegende Studie ist nach Kenntnis der Autoren die erste Studie, die diese Fragestellung untersucht und damit auch eine wissenschaftliche Grundlage für den Diskurs um die Schweigepflicht von Ärzt:innen und Psychotherapeut:innen bereiten kann.

Über die Gründe einer so deutlichen Diskrepanz in der Bewertung der Bedeutsamkeit der Schweigepflicht zwischen Kammern und Fachgesellschaften als Vertretern der Heilberufe und der Allgemeinbevölkerung, die letztlich als potenzielle Patient:innen von einer Lockerung der Schweigepflicht unmittelbar betroffen wären, kann nur spekuliert werden. Die hohe Zustimmung zum Bruch der ärztlichen Schweigepflicht basiert mög- licherweise darauf, dass das Thema von der Bevölkerung als hochsicherheitsrelevant eingeschätzt wird und dabei kaum differenziert wird, ob es sich um eine Gefährdung durch extremistische Gewalt handelt oder ob lediglich eine extremistische Einstellung vorliegt. Wenngleich beide Aspekte auch Überschneidungen aufweisen können (McCauley und Moskalenko 2017), sollte bedacht werden, dass extremistische Einstellungen nicht zwangsläufig mit Gewalttaten verbunden sein müssen oder kausal zusammenhängen, ebenso wenig wie aggressive Fantasien mit Handlungsabsichten gleichzusetzen sind (Allroggen et al. 2012). Die sich daraus ergebende Notwendigkeit für psychologische Psychotherapeut:innen und Ärzt:innen, die Bevölkerung (und potenzielle Patient:innen) stärker auf die Bedeutung und den Stellenwert der Schweigepflicht im Rahmen einer Behandlung aufmerksam zu machen, wird jedoch deutlich, insbesondere im Zusammenhang mit persönlichen (extremistischen) Einstellungen von Patient:innen, die an sich keine Gefährdungslage darstellen.

Zudem zeigen die Daten, dass der Bruch der Schweigepflicht von Psychiater:innen und Psychotherapeut:innen insbesondere dann von der Allgemeinbevölkerung befürwortet wird, wenn die Befragten davon ausgehen, dass eine Verbindung zwischen Terrorismus und psychischen Störungen besteht. Wenngleich diese Vorstellung generell in der Allgemeinbevölkerung weit verbreitet zu sein scheint (dw.com 2020), ist dieser explizite Zusammenhang zwischen psychischen Störungen als Ursache von extremistischer Gewalt wissenschaftlich nicht belegt (Allroggen 2020; Corner et al. 2016; Corner und Gill 2019), sondern differenzierter zu betrachten: Einerseits kann das Vorliegen von einigen psychischen Störungen bei radikalisierten Personen den Übergang in Gewalttaten begünstigen, anderseits kann bei bestimmten psychischen Störungen auch die Übernahme von extremistischen Inhalten erleichtert sein, ohne dass diesen Fällen ein „echter" Radikalisierungsprozess zugrunde liegt. Aufgabe von Psychiater:innen und Psychotherapeut:innen ist es daher auch, über mögliche Zusammenhänge differenzierter zu informieren und über die Bedeutung der Vertraulichkeit der Behand- lung als ihre zentrale Grundlage aufzuklären. Dies ist auch insofern bedeutsam, damit psychisch kranke Menschen weiter die Möglichkeit haben, über aggressive Fantasien im Rahmen der Behandlung zu sprechen und diese ggf. behandeln zu lassen, ohne fürchten zu müssen, dass die Sicherheitsbehörden informiert werden. Dies impliziert aber auch, dass Ärzt:innen und Psychotherapeut:innen entsprechendes Wissen über die Entwicklung von extremistischer Gewalt und im Umgang mit Patient:innen mit extremistischer Einstellung und einer Gefährdungseinschätzung haben.

Interessant ist, dass die Mehrheit der Bevölkerung eine Pflicht zur psychiatrischen bzw. psychotherapeutischen Behandlung von Menschen, die einen Terrorakt planen, sieht. Somit scheint die Meinung vorzuherrschen, dass Psychiater:innen und Psychotherapeut:innen eine bedeutsame Rolle bei der Gefahrenabwendung spielen können - bei gleichzeitig deutlich geringerer Überzeugung, dass eine Behandlung Menschen mit extremistischen Einstellungen grundsätzlich hilft. Möglicherweise zeigt sich damit die implizite Erwartung der Befragten, dass Psychotherapeut:innen und Psychiater:innen eine Art "Wächerfunktion" im Rahmen der Behandlung potenzieller Terrorist:innen übernehmen sollten. Auch hier wäre es Aufgabe, stärker auf die Voraussetzungen für eine gelingende psychotherapeutische/psychiatrische Behandlung hinzuweisen, aber auch auf die Möglichkeiten und Grenzen, die sich im Zusammenhang mit Extremist:innen zeigen können.

\section{Limitationen}

Bei der Betrachtung der Ergebnisse sollte berücksichtigt werden, dass die Befragten direkten Kontakt mit den Interviewer:innen hatten, der selbst dann, wenn der Fragebogen anonym ausgefüllt wurde, die Antwortvergabe im Sinne einer sozial erwünschten Tendenz beeinflusst haben könnte. Zudem war möglicherweise das Thema extremistische Gewalt für viele Studienteilnehmende so abstrakt, dass eine hohe Zustimmung zu den Fragen auch daraus resultieren kann, dass diese sich selbst nicht als potenziell vom Thema Betroffene gesehen haben. Die Fragen waren zu- 
dem sehr allgemein gestellt und erfassen somit eine individuell angenommene Gefährdungslage. In Folgestudien würde sich eine Befragung mit konkreteren Fallvignetten anbieten, mit deren Hilfe differenzierter erhoben werden könnte, zu welchem Zeitpunkt und mit welcher Indikation die Befragten den Bruch der Schweigepflicht und die Einsicht in Patient:innenakten befürworten würden, z. B. wenn bereits Tatpläne bekannt geworden sind o. Ä.

\section{Fazit für die Praxis}

- Kammern und Fachgesellschaften fordern eine konsequente Einhaltung der Schweigepflicht für Ärzt:innen und Psychotherapeut:innen, auch im Zusammenhang mit extremistischen Einstellungen von Patient:innen.

- In einer bevölkerungsrepräsentativen Befragung wurden 2503 Personen zu ihrer Einstellung in Bezug auf die Lockerung der ärztlichen Schweigepflicht bei $\mathrm{Pa}$ tient:innen mit extremistischer Einstellung befragt.

- Für $58 \%$ der Befragten ist der Schutz vor extremistischen Gewalttaten wichtiger als die Einhaltung der ärztlichen Schweigepflicht.

- In der Bevölkerung wird ein Zusammenhang zwischen Terrorismus und psychischen Erkrankungen angenommen und der Bruch der Schweigepflicht insbesondere bei psychischen Erkrankungen befürwortet.

- Aufgabe von Angehörigen der Heilberufe ist es, der Allgemeinbevölkerung die Bedeutung der Schweigepflicht für das Verhältnis zu Patient:innen im Rahmen einer Behandlung stärker zu vermitteln.

\section{Korrespondenzadresse}

\section{Dr. Thea Rau}

Klinik für Kinder- und Jugendpsychiatrie/ Psychotherapie, Universitätsklinikum Ulm Steinhövelstr. 5, 89075 Ulm, Deutschland thea.rau@uniklinik-ulm.de

Danksagung. Die Befragung wurde vom deutschen Markt- und Sozialforschungsinstitut USUMA, BerlinWeißensee, durchgeführt und durch das Bundesamt für Migration und Flüchtlinge (BAMF) im Rahmen des Projekts "Grundlagenwissen für Heilberufe zur Identifikation von Radikalisierungsprozessen als Risiko für Taten zielgerichteter Gewalt" finanziert.

Funding. Open Access funding enabled and organized by Projekt DEAL.

Attitude of the general population to confidentiality of physicians and psychotherapists regarding patients with extremist dispositions

Background: In order to prevent extremist acts of violence, there has been a constant debate on whether the general abolition of medical confidentiality and the confidentiality of psychological psychotherapists concerning patients with extremist attitudes is a useful means of security. So far, there has been no investigation into whether this is a majority argument in the political discourse, as there are no empirical studies on the opinion of the general population in connection with extremist religious or political beliefs and medical confidentiality.

Material and methods: In this study the attitude of the general population was examined by a representative population survey $(n=2503)$.

Results: Over half of the participants (58\%) considered the protection against a possible threat by extremist violence more important than adherence to the confidentiality of psychiatrists and psychotherapists. More than half (54\%) of the participants supported the statement that it should be principally possible to break the medical confidentiality if the physician or psychotherapist gained knowledge of the extremist attitude of the patient. Approval was explicitly higher in cases in which the participants saw an association between terrorism and psychiatric diseases.

Conclusion: The results showed that it is important to enlighten the general population about the necessity of medical confidentiality as a central principle of a curative treatment but it is also crucial to take a very critical view of what role mental illness actually plays in the emergence of extremist violence in order to avoid stigmatization of those affected.

\section{Keywords}

Terrorism · Mental illness · Professional-patient relations · Stigmatization · Population representative survey

\section{Einhaltung ethischer Richtlinien}

Interessenkonflikt. T. Rau, S. Mayer, J.M. Fegert und M. Allroggen geben an, dass kein Interessenkonflikt besteht.

Alle beschriebenen Untersuchungen am Menschen oder an menschlichem Gewebe wurden mit Zustimmung der zuständigen Ethikkommission, im Einklang mit nationalem Recht sowie gemäß der Deklaration von Helsinki von 1975 (in der aktuellen, überarbeiteten Fassung) durchgeführt. Von allen Studienteilnehmenden liegt eine Einverständniserklärung vor.

Open Access. Dieser Artikel wird unter der Creative Commons Namensnennung 4.0 International Lizenz veröffentlicht, welche die Nutzung, Vervielfältigung, Bearbeitung, Verbreitung und Wiedergabe in jeglichem Medium und Format erlaubt, sofern Sie den/die ursprünglichen Autor(en) und die Quelle ordnungsgemäßnennen, einen Link zur Creative Commons Lizenz beifügen und angeben, ob Änderungen vorgenommen wurden.

Die in diesem Artikel enthaltenen Bilder und sonstiges Drittmaterial unterliegen ebenfalls der genannten Creative Commons Lizenz, sofern sich aus der Abbildungslegende nichts anderes ergibt. Sofern das betreffende Material nicht unter der genannten Creative Commons Lizenz steht und die betreffende Handlung nicht nach gesetzlichen Vorschriften erlaubt ist, ist für die oben aufgeführten Weiterverwendungen des Materials die Einwilligung des jeweiligen Rechteinhabers einzuholen.
Weitere Details zur Lizenz entnehmen Sie bitte der Lizenzinformation auf http://creativecommons.org/ licenses/by/4.0/deed.de.

\section{Literatur}

\section{Verwendete Literatur}

Allroggen M (2020) Psychische Störungen im Zusammenhang mit Radikalisierung. https://www.bpb.de/politik/extremismus/ radikalisierungspraevention/306897/psychischestoerungen-im-zusammenhangmit-radikalisie rung.Zugegriffen: 5. Nov. 2020

Allroggen M, Kliemann A, Spröber N, Rau T, Fegert JM (2012) Bedrohungsszenarien und Gewalt gegenüber Dritten in der Psychotherapie. Psychotherapeut 57(2):142-147

Bühring P (2016) Radikalisierung: „Die Ideologien verschreiben Orientierung und Halt". Dtsch Arztebl 113(14):A-634/B-536/C-532

Bühring P (2018) Islamistisch motivierte Radikalisierung. Die innere Sicherheit. Dtsch Arztebl Int 115(23):A-1114/B-937/C-933

Corner E, Gill P (2019) Psychological distress, terrorist involvement and disengagement from terrorism: a sequence analysis approach. J Quant Criminol 36:499-526

Corner E, Gill P, Mason O (2016) Mental health disorders and the terrorist: a research note probing selection effects and disorder prevalence. Stud Confl Terror 39:560-568 
Dom G, Schouler-Ocak M, Bhui K et al (2018) Mass violence, radicalization and terrorism: a role for psychiatric profession? Eur Psychiatry 49:78-80 https://taz.de/Messerangriff-von-Wuerzburg/! 5782038/.Zugegriffen:21. Juli 2021

https://www.aerzteblatt.de/nachrichten/69968/ Montgomery-warnt-vor-Lockerung-deraerztlichen-Schweigepflicht. Zugegriffen: 5 . Nov. 2020

https://www.aerztezeitung.de/Politik/Lockerungder-Schweigepflicht-vom-Tisch-311744.html. Zugegriffen: 19. März 2021

https://www.bayerische-staatszeitung.de/staatszei tung/politik/detailansicht-politik/artikel/cduund-csu-ruetteln-an-der-aerztlichen-schweigepflicht.html\#topPosition. Zugegriffen: 21. Juli 2021

https://www.bptk.de/wp-content/uploads/2019/01/ 20060113_musterberufsordnung.pdf. Zugegriffen:28. Okt. 2021

https://www.bundesaerztekammer.de/fileadmin/ user_upload/downloads/pdf-Ordner/MBO/ MBO-AE.pdf.Zugegriffen:28.Okt. 2021

https://www.dw.com/de/im-namen-der-sicherheit/ a-19468650.Zugegriffen:5.Nov. 2020

https://www.handelsblatt.com/politik/deutschland/ terrorbekaempfung-auslaendische-gefaehrderschneller-abschieben/13991964-2.html? ticket=ST-7447319-GaiYowkE3Irco9tB4jT3ap3.Zugegriffen: 19. März 2021

https://www.psychotherapeutenjournal.de/ptk/web. nsf/gfx/B8FED94FEA0991C6C1257E660027C642/ \$file/ptj_2015-2.pdf.Zugegriffen:28.Okt.2021

https://www.sueddeutsche.de/politik/terrorradikalisierung-schizophrenie-schweigepflicht1.4385054.Zugegriffen: 3. März 2021

https://www.tagesschau.de/inland/hanau-investigativ101.html.Zugegriffen:5.Nov. 2020

Klett M, Tessmer S (2016) Lockerung der Schweigepflicht zum Zweck der Verhinderung von Straftaten? Psychother J 4:380-386

Linden M, Helmchen H (2018) Ethische Problemstellungen in der Psychotherapie. Psychotherapeut 63:68-74

McCauley C, Moskalenko (2017) Understanding political radicalization: The two-pyramids model. Am Psychol 72(3):205-2016. https://doi. org/10.1037/amp0000062

Parzeller M, Wenk M, Rothschild M (2005) Die ärztliche Schweigepflicht. Dtsch Arztebl 102(5):A-289/B237/C-224

Schomerus G, Stolzenburg S, Bauch A, Speerforck S, Janowitz D, Angermeyer MC (2017) Shifting blame? Impact of reports of violence and mental illness in the context of terrorism on population attitudes towards persons with mental illness in Germany. Psychiatry Res 252:164-168

Weltärztebund (2013) WMA Deklaration von Helsinki - Ethische Grundsätze für die medizinische Forschung am Menschen. https://www. bundesaerztekammer.de/fileadmin/user upload/downloads/pdf-Ordner/International/ Deklaration_von_Helsinki_2013_20190905. pdf.Zugegriffen:2 Dez 2021

ICC/ESOMAR (2007) International code on market and social research. https://iccwbo.org/ content/uploads/sites/3/2008/01/ESOMARINTERNATIONAL-CODE-ON-MARKET-ANDSOCIAL-RESEARCH.pdf. Zugegriffen: 2 Dez 2021

\section{Weiterführende Literatur}

Grundgesetz für die Bundesrepublik Deutschland in der im Bundesgesetzblatt Teil III, Gliederungsnummer 100-1, veröffentlichten bereinigten Fassung, das zuletzt durch Artikel 1 u. 2 Satz 2 des Gesetzes vom 29. September 2020 (BGBI.IS 2048) geändert worden ist

Gesetz zur Kooperation und Information im Kinderschutz vom 22. Dezember 2011 (BGBI. IS. 2975) das zuletzt durch Artikel 2 des Gesetzes vom 3. Juni 2021 (BGBI.IS. 1444) geändert worden ist

Strafgesetzbuch in der Fassung der Bekanntmachung vom 13. November 1998 (BGBI. I S. 3322), das zuletzt durch Artikel 3 des Gesetzes vom 8. Oktober 2021 (BGBI. I S. 4650) geändert worden ist

\section{Zu Artikel: Rau et al. (2021)}

In dem Artikel von Rau, Mayer, Fegert und Allroggen zur "Einstellung der Allgemeinbevölkerung zur Schweigepflicht von Ärzt:innen und Psychotherapeut:innen bei Patient:innen mit extremistischer Einstellung" im Psychotherapeut (https://doi.org/10.1007/s00278-021-00558-7; November 2021) wird geschrieben: Bereits 2016 wurden im Zusammenhang mit Attentaten und in der Folge der Verschärfung des Antiterrorgesetzes eine Beteiligung von Ärzt:innen und Psychotherapeut:innen an der Terrorbekämpfung gefordert und auch die Lockerung der Schweigepflicht diskutiert (tagesschau.de 2020; Linden und Helmchen 2018; dw.com 2020).

Das Zitat könnte den Eindruck wecken, als würden wir für eine Lockerung der Schweigepflicht sprechen. Das Gegenteil ist der Fall. In der zitierten Arbeit haben wir auf ethische Probleme in der Psychotherapie hingewiesen und bzgl. der Schweigepflicht und Vertraulichkeit in der Beziehung als Probleme auf die computerisierte Weitergabe von Fallberichten an Behörden hingewiesen, auf sozialrechtliche und strafrechtliche Offenbarungspflichten, auf die Abwägung zwischen konkurrierenden Rechtsnormen, auf die Gruppenpsychotherapie. Wir haben deutlich gemacht, dass die Schweigepflicht an vielen Stellen in Gefahr ist und dass „einige dieser Problemstellungen Psychotherapeuten persönlich in erhebliche Probleme bringen können", sie sich aber dennoch im Interesse der Patienten gegen übergriffige Anforderungen stellen müssen, da "die Schweigepflicht in keinem anderen Bereich der Heilkunde so bedeutungsvoll, aber auch so kompliziert ist, wie in der Psychotherapie".

Wir haben also nicht für eine Lockerung der Schweigepflicht votiert, wie das o.g. Zitat nahelegen könnte, sondern für eine Sicherung der Schweigepflicht, sogar dann, wenn dies Probleme für den Therapeuten mit sich bringt.

Autoren: M. Linden \& H. Helmchen 